\title{
Aplicación alternada de ácido acetilsalicílico con fungicidas en el control de mildeo polvoso en rosa
}

\section{Acetylsalicylic acid with fungicides, as an alternative application, for the powdery mildew in rose's control}

Fecha de Recepción: 14 de marzo de 2013

Fecha de Aceptación: 18 de octubre de 2013
Sindy Paola Torres Velásquez¹, Jorge Velandia Monsalve², Héctor Murcia Herrera ${ }^{3}$

\section{Resumen}

Objetivo. Evaluar el efecto de aplicaciones de ácido acetilsalicílico (AAS) alternadas con fungicidas en el control de Mildeo polvoso (Sphaerotheca pannosa) en rosa. Materiales y métodos. Se utilizó el diseño experimental completamente al azar, con 4 repeticiones por tratamiento, y un testigo absoluto; los tratamientos fueron: aplicación de AAS alternada con una de fungicida, dos aplicaciones de AAS alternadas con una de fungicida, tres aplicaciones de AAS alternadas con una de fungicida, aplicaciones de solo fungicidas y aplicaciones de solo AAS. Se evaluó la incidencia y severidad de la enfermedad, la producción, \% de tallos exportables y relación costo-beneficio. Resultados. Se determinó menor incidencia y severidad de Mildeo polvoso realizando una aplicación de AAS alternada con una de fungicida, y tres aplicaciones consecutivas de AAS alternadas con una de fungicida. En la producción, el número promedio de tallos cosechados por mes fue mayor en el testigo absoluto y con tres aplicaciones

\begin{abstract}
Objective. To evaluate the Acetylsalicylic Acid (ASA) effect in the Sphaerotheca pannosa control, which causes Powdery Mildew in the roses cultivation, one of the export flowers' major constraints in production and quality. Materials and methods. In the present investigation, is considered the Acetylsalicylic Acid (ASA) application effect, alternating with fungicides. The treatments consisted of: 1) An absolute control, 2) A AAS application, alternated with a fungicide, 3) Two applications of AAS alternating with a fungicide, 4) Three applications of AAS alternating with a fungicide, 5) Fungicide-only applications and 6) ASA-only applications. We used a completely randomized design with 4 replicates. Results. It showed a powdery mildew disease lower incidence and severity in in the production of exportable stems and cost benefit, by AAS application, alternated with a fungicide and three AAS consecutive applications. The average number production of stems harvested per month,
\end{abstract}

1 Ingeniera Agrónoma. Profesional Independiente. sptorres18@live.com

2 Ingeniero Agrónomo M.Sc. Docente Programa de Ingeniería Agronómica. Universidad de los Llanos. jvelandi@hotmail.com

3 Ingeniero Agrónomo. Asesor Técnico Independiente. hectormurciap@hotmail.com 
de AAS alternadas de una de fungicida, sin diferencias estadísticas en comparación con los demás tratamientos. El porcentaje de tallos de exportación fue mejor con tres aplicaciones de AAS alternadas de una aplicación de fungicida, superando el testigo y el tratamiento con solo fungicidas, presentándose diferencias significativas $(p<0,001)$, y se obtuvo la mayor relación costobeneficio. Conclusión. Aplicaciones del ácido acetilsalicílico alternadas con fungicida son una alternativa que permite reducir la frecuencia de aplicaciones de fungicidas en el cultivo de rosas para el control de Mildeo polvoso.

Palabras clave: Mildeo polvoso, ácido acetilsalicílico, cultivo de rosas (Fuente: USDA). was higher, in absolute control and AAS with three fungicide alternating applications. No statistical differences compared with other treatments were found. The export stems percentage, was better with three applications of AAS alternating fungicide application than the control and treatment with fungicides only significant differences $(p<0.001)$ and had the highest cost-benefit ratio. Conclusion. Applications of Acetylsalicylic Acid alternating with a fungicide is an alternative that reduces the frequency of fungicide applications in the cultivation of roses for Powdery Mildew control.

Key words: Sphaeroteca pannosa, acetyl salicylic acid, rose production (Source: USDA). 


\section{Introducción}

Las flores constituyen el segundo producto agrícola más importante de Colombia; el 98\% de su producción se exporta, haciendo que el país se posicione como el segundo exportador mundial de flores frescas cortadas, con una participación de $14 \%$ en el comercio total, después de Holanda, que cuenta con una participación del $56 \%$. El cultivo de rosa tiene una participación de $29 \%$ de las exportaciones totales (1). Debido a la alta exigencia de calidad por el consumidor de rosas y a la gran competitividad en el mercado, es necesario ofrecer una flor libre de daños causados por agentes fitopatógenos, que afectan su presentación.

Mildeo polvoso (Sphaeroteca pannosa) es una enfermedad limitante en el cultivo de rosa bajo invernadero, la cual causa pérdidas significativas en la productividad de flor de corte (2); para su control, C.I. Exotic Farms S.A ha venido realizando una aplicación semanal de fungicidas preventivos o curativos, y en algunos casos hasta cinco días entre aplicaciones; esto implica un costo alto en el manejo de este fitopatógeno. Se ha reportado el control de varias enfermedades en algunas especies con la aplicación de ácido acetilsalicílico, sin embargo, no se ha utilizado para el control de Mildeo polvoso en el cultivo de rosa $(3,4)$. A fin de buscar alternativas para el control eficiente de este hongo en rosa (Rosa spp var. Clasicc Cezane), con productos económicos y de fácil adquisición, este trabajo de investigación evaluó el efecto de la aplicación alternada del ácido acetilsalicílico con fungicida en cultivos de esta especie bajo condiciones de invernadero, partiendo de la hipótesis de que el ácido acetilsalicílico puede estimular el mecanismo de defensa de la planta y ser una herramienta que permita el control eficiente de este hongo y, por lo tanto, la obtención de plantas sanas, con mayor rendimiento y rentabilidad, al disminuir las aplicaciones de productos químicos.

\section{Materiales y métodos}

Sitio de estudio. El trabajo se desarrolló en la finca Lomas, de $\mathrm{Cl}$. Exotic Farms S.A., ubicada en la vereda La Fuente, del municipio de Tocancipá, Cundinamarca, con coordenadas $4^{\circ} 55^{\prime}$ latitud Norte y $74^{\circ} 06^{\prime}$ al Oeste de Greenwich, con una altura de 2569 msnm y temperatura promedio de $14{ }^{\circ} \mathrm{C}$.
Metodología experimental. La variedad que se utilizó fue Classic Cezane, sembrada bajo cubierta en camas de $32 \mathrm{~m}$ de largo y $0.70 \mathrm{~m}$ de ancho. Se evaluó la frecuencia de aplicaciones del ácido acetilsalicílico $(0.3 \mathrm{gr} / \mathrm{l})$. Las aplicaciones se hicieron cada semana, alternadas con el fungicida, durante dos y tres semanas. En las aplicaciones del fungicida se utilizaron fungicidas protectantes y sistémicos como Triflumizole, Bupirimato, Tebuconazole+Triadimenol, Mancozeb, Dodemorf Acetato y Kresoxim Metil, entre otros, con el fin de reducir el riesgo de resistencia de Oidium en el cultivo de rosas; de cada uno de los fungicidas se utilizó la dosis recomendada por la casa comercial.

La variable incidencia se determinó mediante observación de la presencia o no del hongo en las hojas de las plantas-muestra, anotando el porcentaje que le correspondía a cada muestra; se evaluó cada 8 días, desde el inicio de las aplicaciones de cada tratamiento hasta el final. La variable severidad consistió en determinar el porcentaje por muestra de acuerdo con el porcentaje del tejido afectado por el hongo; se evaluó cada 8 días desde el inicio de las aplicaciones de cada tratamiento hasta el final. Para determinar la producción por tratamiento, se evaluó la variable producción, que consistió en el conteo directo del número de tallos producidos por tratamiento y por mes; esta evaluación se realizó desde el inicio de producción hasta el final.

Con respecto al efecto de cada tratamiento sobre la calidad de la flor, se determinó el número de flores tipo exportación y nacional de acuerdo con los parámetros de clasificación establecidos por la finca; consistió en clasificar todos los tallos producidos por cada tratamiento en la sala de poscosecha. Para conocer la eficiencia agronómica del tratamiento más favorable en cuanto a las aplicaciones de ácido acetilsalicílico y fungicida, se determinó el costo de cada uno de los tratamientos, con base en el precio de cada producto, la dosis, costo/dosis, la cantidad de litros por cama para cada aplicación y el número de aplicaciones; luego, se descontó este costo del valor total de la producción en tallos de exportación, y finalmente se obtuvo el beneficio económico de cada tratamiento. 
Diseño experimental. Se empleó un diseño experimental completamente al azar, con seis tratamientos y cuatro repeticiones por tratamiento. Cada tratamiento tuvo 4 repeticiones, para un total de 24 unidades experimentales. Una unidad experimental corresponde a cada cama estándar de producción. Las aplicaciones se hicieron durante 12 semanas -una aplicación por semana, dependiendo de la rotación de productos de la finca-, y la evaluación, durante 16 semanas. Los datos obtenidos se analizaron con las pruebas estadísticas de ANAVA y Tukey (\%), utilizando el programa estadístico SPSS11.5.

\section{Resultados y discusión}

Incidencia de Mildeo polvoso en rosa var Classic Cezane. Los resultados del ANAVA mostraron efectos estadísticos de los tratamientos en el control de la incidencia y severidad del Mildeo polvoso en rosa. En el testigo sin aplicaciones, los primeros síntomas de la enfermedad se presentaron a partir de la tercera semana del experimento, y con la aplicación de los tratamientos, en la quinta semana.

Con una aplicación de ácido acetilsalicílico (AAS) alternada con una de fungicida, la incidencia de la enfermedad fue menor en comparación con la obtenida con el testigo sin aplicación; con dos aplicaciones consecutivas de AAS alternadas con una de fungicida, desde la semana 6 hasta la semana 12, y con aplicación de solo AAS desde la semana 8 hasta la semana 12 se obtuvieron diferencias estadísticas significativas $(p<0,005)$, y con los tratamientos de tres aplicaciones de AAS y solo fungicida no se encontraron diferencias significativas en el control de la incidencia de la enfermedad. Con las aplicaciones de solo fungicida la incidencia de la enfermedad fue menor en comparación con la obtenida con aplicaciones de solo AAS, sin presentar diferencias estadísticas desde la semana 4 hasta la semana 12 .

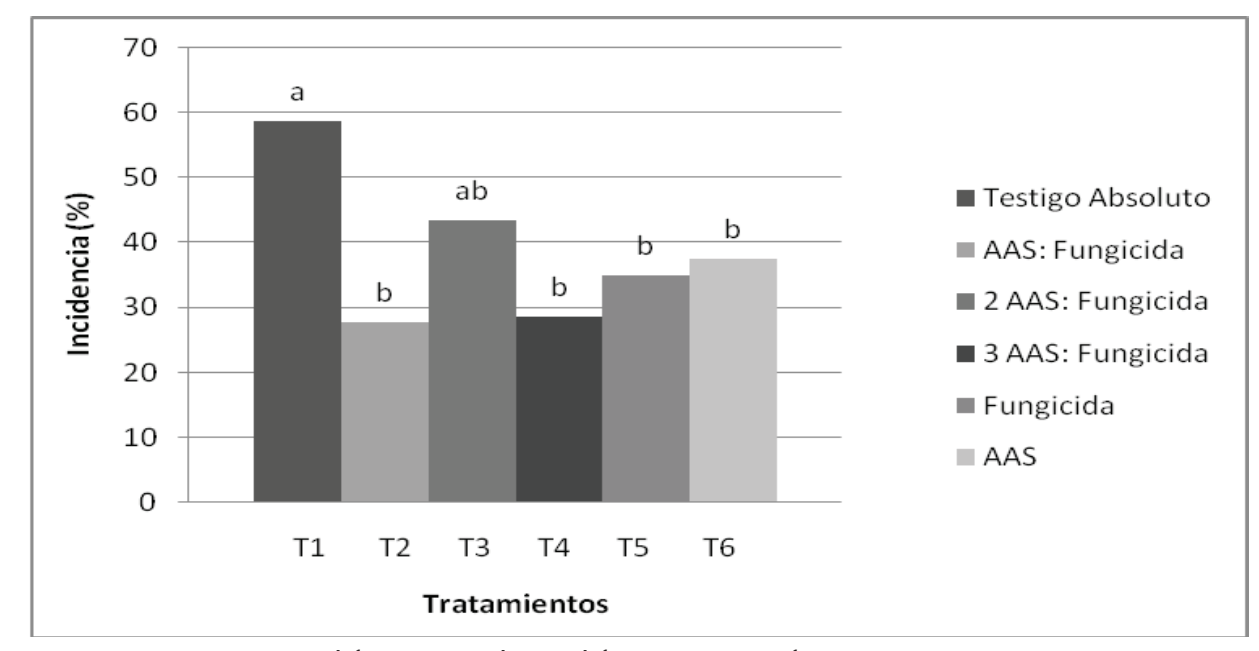

Letras diferentes indican diferencias significativas $(p<0.05)$

FIGURA 1. EFECTO DE LOS TRATAMIENTOS EN EL CONTROL DEL MILDEO POLVOSO EN ROSA VAR CLASSIC CEZANE

De acuerdo con Umetamy et al. (1990), las aplicaciones exógenas de AAS promueven una reducción de la incidencia de enfermedades, ya que este tiene el efecto de activador de resistencia, lo que concuerda con los resultados obtenidos en esta variable. Por otra parte, Tally et al. (1999) señalan que el AAS es altamente móvil dentro de la planta, por eso puede proteger hasta partes no cubiertas en la aplicación foliar, o se puede aplicar por el sistema de riego, para ser absorbido por el sistema radicular. 
Severidad de Mildeo polvoso en rosa var. Classic Cezane. El ANAVA mostró efectos estadísticos de los tratamientos en el control de la severidad del ataque de Mildeo Polvoso. El ataque de Mildeo Polvoso fue menor con una aplicación de AAS alternada con una de fungicida, seguida de tres aplicaciones de AAS con una de fungicida, en comparación con el testigo, con diferencias estadísticas al $5 \%$ desde la semana 4 hasta la semana 12 de aplicación.

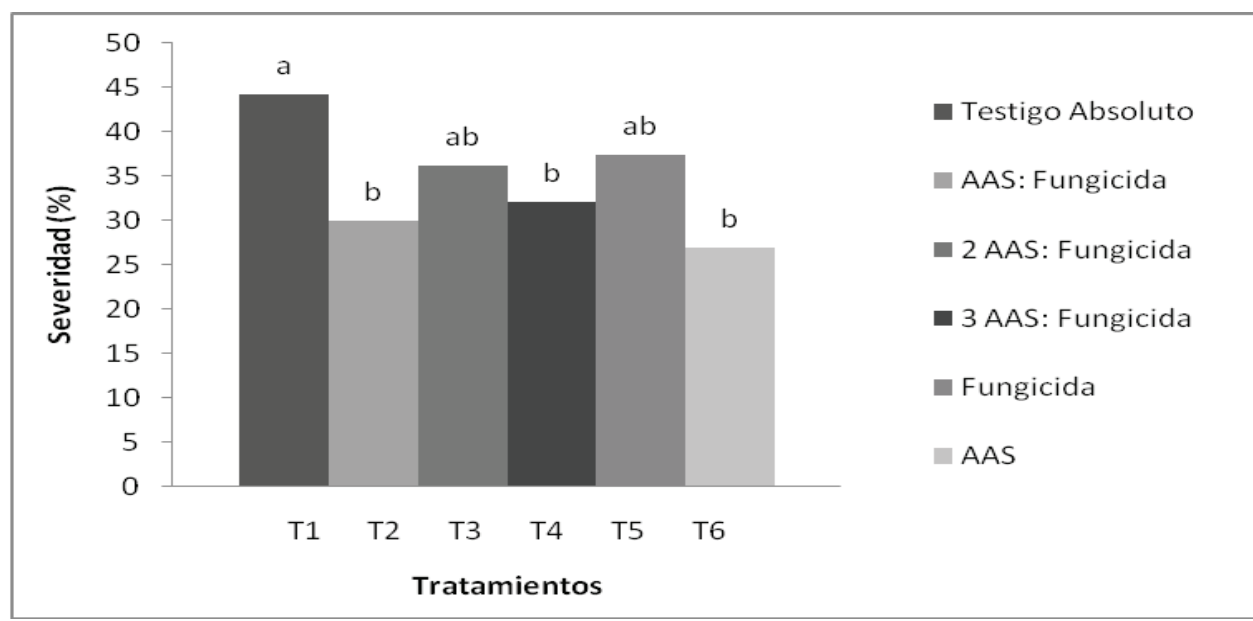

Letras diferentes indican diferencias significativas $(p<0.05)$

FIGURA 2. EFECTO DE LOS TRATAMIENTOS EN EL CONTROL DE LA SEVERIDAD DE MILDEO POLVOSO EN ROSA VAR CLASSIC CEZANE

Desde la semana 5 hasta la semana 8 , las aplicaciones de AAS superaron las aplicaciones de solo fungicida en el control de la severidad de la enfermedad, sin presentar diferencias estadísticas entre sí, y fueron mejores desde la semana 9 hasta la semana 12, con diferencias estadísticas entre sí, posiblemente, debido a la generación de resistencia del hongo a los fungicidas que se utilizan para su control. De acuerdo con Serres et al. (1976), la resistencia de los hongos a productos químicos constituye un punto esencial en la presencia de determinadas razas del hongo; este fenómeno se manifiesta por una reducción de la sensibilidad a los productos químicos, y esas características se transmiten genéticamente (7).

Con los demás tratamientos, la severidad de Mildeo polvoso presentó valores intermedios a los registrados con una aplicación de AAS alternada con una aplicación de fungicida, con dos aplicaciones de AAS alternadas con una de fungicidas y con aplicaciones de solo AAS. De acuerdo con Radman et al. (2003), las plantas muestran las respuestas fisiológicas y morfológicas a una serie de factores físicos y químicos conocidos como inductores, lo que explica que la aplicación de AAS como inductor genera activación del mecanismo de resistencia en las plantas. Así también, Sakamoto et al. (1999) obtuvieron en sus investigaciones en arroz la formación de resistencia adquirida contra el añublo de la vaina (Pyricularia oryzae).

\section{Determinación de producción por tratamiento.} Con respecto a la producción, se aprecia un mayor número de tallos cosechados por mes en el testigo $(575,2)$, seguido de tres aplicaciones de AAS alternadas con una de fungicida (573), sin diferencias estadísticas entre sí, y fueron mejores en comparación con el obtenido con dos aplicaciones de AAS alternada con una de fungicida, aplicaciones de solo fungicida y solo AAS, con diferencias estadísticas altamente significativas. Estos resultados están de acuerdo con lo expresado por Shah y Klessig (1995), quienes reportaron que la aplicación de pesticidas obstruye parcialmente los estomas, afectando la fotosíntesis y la tasa de producción de brotes. 


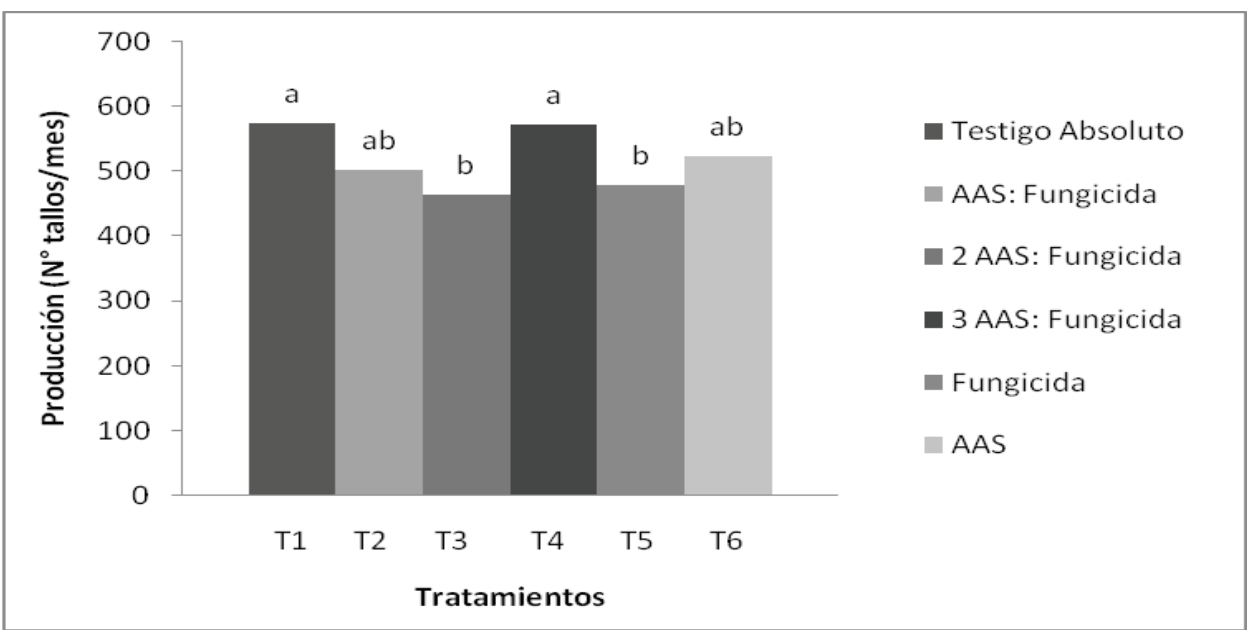

Letras diferentes indican diferencias significativas $(p<0.05)$

FIGURA 3. EFECTO DE LOS TRATAMIENTOS EN LA PRODUCCION DE ROSA (ROSA VAR CLASSIC CEZANE)

Tallos exportables. El mayor porcentaje de tallos exportables se dio con tres aplicaciones de AAS alternadas con una de fungicida $(96,2 \%)$, seguido de una aplicación de AAS y una de fungicida y aplicaciones de solo AAS, sin diferencias estadísticas entre sí, y fueron mejores en comparación con el testigo (35\%) y con aplicaciones de solo fungicida. Las aplicaciones de solo fungicida presentaron bajo porcentaje de tallos exportables, como consecuencia del menor efecto en el control de la enfermedad.

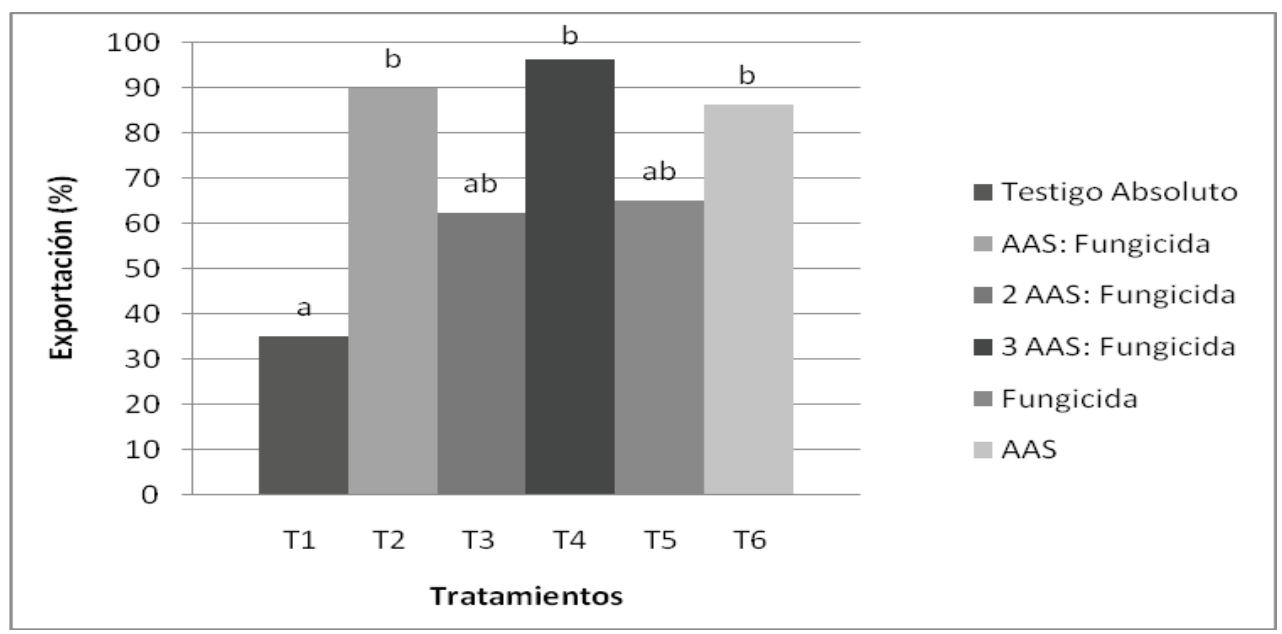

Letras diferentes indican diferencias significativas $(p<0.05)$

FIGURA 4. EFECTO DE LA APLICACIÓN DE AAS ALTERNADAS CON FUNGICIDAS SOBRE EL PORCENTAJE DE TALLOS DE EXPORTACIÓN 


\section{Referencias bibliográficas}

(1) Asocolflores. Estadísticas (en línea) 2007 (fecha de acceso 10 de agosto 2012). URL disponible en http://www.asocolflores. org/info/info.php.

(2) Umetamy RA, Deverall BJ. Studies on crossprotection in the anthracnose disease of bean. Physiol Plant Pathol 1990; 2: 357-374.

(3) Raskin I. Role of salicilic acid in plants. Rev Plant Physiol 1992; 43: 439-463.

(4) Hoog J. Handbook for moderm greenhouse rose cultivation. Appl Plant Res 2001; 8(2): 219-225.

(5) Tally DF. Active oxygen species in the induction of plant systemic acquired resistance by salicylic acid. Science 1999; 262:1883-1886.

(6) Serres $P$, Seskar $M$, Kanter D, Schweizer $P$, Metraux JP. Salicylic acid in rice. Plant Physiol 1976; 108: 633-39.
(7) Klessig DF, Malan EM. Active oxygen species in the induction of plant systemic acquired resistance by salicylic acid. Science 1994; 262: 1883-1886.

(8) Radman C. The search for proteinase inhibitorinducing factor, PIIF. Plant Mol Bio 2003; 19: 123-133.

(9) Sakamoto Y, Kodakary E, Yamamura T, Tanaka S, Tabata, M. Glucosylation of salicilic acid by cell suspensión cultures of Mallatus japonicus. Plant Cell Reports 1999; 9: 325- 327.

(10) Shah J, Klessig DF. Identification of a salicylic acid-responsive element in the promoter of the tobacco pathogenesis-related $\beta$ 1,3-glucanase gene, PR-2d. Plant J 1995; 10: 1089- 1101. 